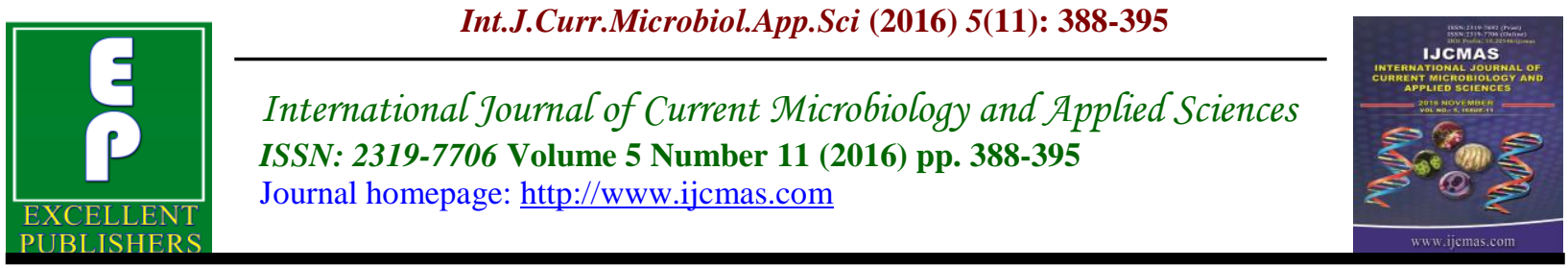

Original Research Article

http://dx.doi.org/10.20546/ijcmas.2016.511.044

\title{
Assessment of Amp C Beta-lactamase Production by Various Methods in Urinary Isolates of Escherichia coli from a Tertiary Care Teaching Hospital
}

\author{
Anandhi Lakshmanan ${ }^{1}$, Dharmalingam Thirunavukkarasu ${ }^{2} *$, T.Umaarasu ${ }^{3}$ and S. Rajesh ${ }^{2}$ \\ ${ }^{1}$ Department of Microbiology, ESIC Medical College and PGIMSR, Chennai, India \\ ${ }^{2}$ Department of Microbiology, Government Mohan Kumaramangalam Medical College, \\ Salem, Tamil Nadu, India \\ ${ }^{3}$ Department of Microbiology, Vivekananda Dental College for Women, Tiruchengode, \\ Namakkal, Tamil Nadu, India \\ *Corresponding author
}

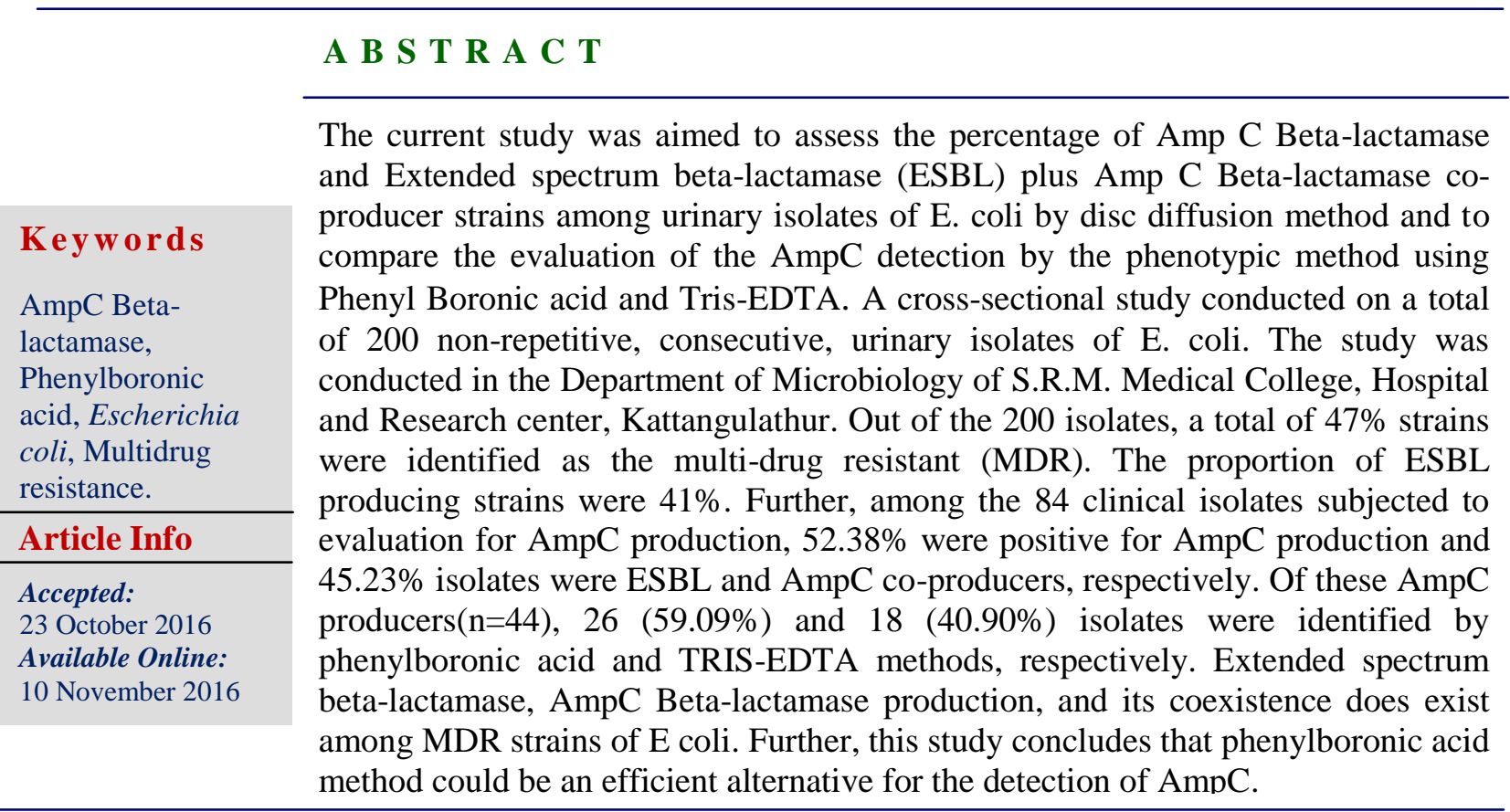

\section{Introduction}

The production of $\beta$-lactamase is the principal mechanism by which Gramnegative bacteria resist the action of $\beta$ lactam antibiotics (Paniagua-Contreras et al., Sood et al., 2012). In recent decades, there has been an alarming rise in infections caused by bacteria that express extendedspectrum $\beta$-lactamases (ESBLs) (Sood et al.,
2012; Mittal et al., 2014). Many Enterobacteriaceae organisms also produce broad-spectrum cephalosporinases (AmpC) by intrinsic chromosomally encoded mechanism (Harris et al., 2015). But recently, high-level Amp C AmpC production is also increasingly being reported in new species like Escherichia coli via plasmid acquisition (Jaurin et al., 1981; 
Tracz et al., 2007). This Plasmid-mediated Plasmid-mediated Amp C AmpC can coexist with ESBL enzymes in the same host (Taneja et al., 2008; Tracz et al., 2007). These ESBL and Amp C AmpC coproducers pose a special challenge to susceptibility testing as they make interpretation of various assessment methods less reliable and increases the probability of false susceptibility reporting to betalactamases (Harris et al., 2015; Paltansing et al., 2015; Yang et al., 2007).

Even though various laboratory methods have been evolved in recent years, the validity and reliability of these methods are quite variable. No standard guidelines have evolved to recommend any particular method in a given setting (Manoharan et al., 2012; Hassan et al., 2013). Considering the wide geographical variation in the occurrence of Amp C AmpC production and resource availability, it is of vital importance to identify simple, quick, valid and c costeffective laboratory methods suitable for resource-limited resource-limited settings like India (Tracz et al., 2007; Ahmed et al., 2015).

Several method shave methods have been proposed to assess the Amp C AmpC production by microorganisms. These methods broadly include disc diffusion methods, agar based agar-based methods like cefoxitin agar method (Gupta et al., 2012; Handa et al., 2013). phenotypic inhibitor based methods like phenyl boronic acid (Hassan et al., 2013; Sageerabanoo et al., 2015; Shanthi et al., 2012). and the three-dimensional extraction test (3DET) (Kumar et al., 2014).

The validity and reliability of these tests are quite variable and often put the clinical microbiologist in a dilemma in recommending appropriate anti-microbial agents key $\beta$-lactam resistant isolates, especially third-generation cephalosporins (Drinkovic et al., 2015).

Considering the lack of proper recommendations and scarcity of the studies in this vital area and also the ever increasing incidence of multidrug-resistant multidrugresistant strains of $E$. coli, the present study has been undertaken to fill this knowledge gap.

\section{Materials and Methods}

The study was a cross-sectional study conducted on a total of 200 non repetitive non-repetitive, consecutive, urinary isolates of $E$. coli. The study was conducted in the Department of Microbiology of S.R.M. Medical College, Hospital and Research centre, Kattangulathur. The study was conducted between the period of January 2011 to January 2012. Initially, all the samples were subjected to wet mount and Gram Staining. Then samples were cultured on Cysteine Lactose Electrolyte Deficient Agar (CLED) and standard biochemical tests were done for identification of $E$. coli. The identified isolates were subjected to antibiotic susceptibility testing as per CLSI guidelines using ATCC E. coli 25922 strain.

All strains, which were resistant to the second generation Cephalosporin were screened for extended spectrum betalactamase (ESBL) production by disk diffusion method using Cefoxitin $(30 \mu \mathrm{g})$, Ceftriaxone $(30 \mu \mathrm{g})$, Ceftazidime $(30 \mu \mathrm{g})$, Ceftazidime/Clavulanic acid $(30 / 10 \mu \mathrm{g})$, on Mueller Hinton Agar. The reference zone for Ceftriaxone $<25 \mathrm{~mm}$, for Ceftazidime $<22 \mathrm{~mm}$ and $\leq 27 \mathrm{~mm}$ for Ceftazidime/Clavulanic acid, according to CLSI guidelines. 
All the second generation Cephalosporin resistance isolates were screened for Amp C, beta-lactamase disk diffusion method using Cefoxitin (30 $\mu \mathrm{g})$, Ceftazidime $(30 \mu \mathrm{g})$, Ceftazidime/Clavulanic acid (30/10 $\mu \mathrm{g})$. The diameter of a zone of inhibition was noted and interpreted according to the CLSI guidelines. All these strains were also assessed for ESBL and Amp C coproduction by disc diffusion method.

All isolates, which were shown to be Amp C producers in disc diffusion method were assessed for Amp C production by the phenotypic method, using phenyl boronic phenylboronic acid. Peptone water suspension of clinical isolates equivalent to $0.5 \mathrm{Mc}$ Farland Standard was prepared. A sterile swab was dipped into the suspension and inoculated (lawn culture) on the Mueller-Hinton Mueller-Hinton Agar was made. Plate surface was allowed to dry.

A disk containing $30 \mu \mathrm{g}$ of Cefoxitin and another Cefoxitin disc $(30 \mu \mathrm{g})$ impregnated with $400 \mu \mathrm{g}$ of Phenyl Boronic Phenylboronic Acid were placed on the agar. Plates were incubated at $37^{\circ} \mathrm{C}$ for 18 24 hours. Isolates demonstrating a zone diameter around the disk containing Cefoxitin and Boronic Acid $\geq 5 \mathrm{~mm}$ than the zone diameter around the disk containing Cefoxitin alone were considered an as Amp $\mathrm{C}$ producer.

Subsequently, Amp C producing isolates were assessed for Amp C production by using Tris EDTA-Amp C disk test. A Lawn culture of E. coli ATCC 25922 was prepared on Mueller Hinton Agar plate. Sterile disk $(6 \mathrm{~mm})$ was moistened with Tris-EDTA TrisEDTA $(20 \mu \mathrm{l})$ and inoculated with several colonies of the test organism.

The inoculated disk was then placed beside a Cefoxitin disk (almost touching) on the inoculated plate. The plates were incubated at $35^{\circ} \mathrm{C}$ for $18-24 \mathrm{Hrs}$. A positive test appeared as the flattening or indentation of the Cefoxitin inhibition zone.

\section{Results and Discussion}

A total of 200 isolates of Escherichia coli which were consecutive, non-duplicate samples isolated were included in the study. The age distribution of isolates showed that all age groups are being affected by $E$. coli. The proportion of participants in the paediatric age group (below 15 years) was $6 \%$, in adolescent and adult age group up to 45 years was $49.5 \%$. About $2.5 \%$ of participants were between 46 to 60 years and the remaining 225 were above 60 years.

The proportion of females (73\%) was much higher than the proportion of males $(27 \%)$ (Table 1).

Out of the 200 isolates, the proportion of multidrug resistance strains) resistant to third generation cephalosporin was $47 \%$ (95\% CI $40.08 \%$ to $53.92 \%$ ). The proportion of ESBL producing strains was $41 \%(95 \%$ CI $34.18 \%$ to $47.82 \%$ ) (Table 2).

Among the 84 clinical isolates were subjected to evaluation of Amp C production by disc diffusion method, $52.38 \%$ (95\% CI $41.7 \%$ and $63.06 \%$ ) were positive for Amp C production and 45.23\% (95\% CI $34.59 \%$ to $55.87 \%$ ) isolates were ESBL and Amp C co-producers (Table 3).

All the 44 Amp $C$ producers by disc diffusion method were evaluated by phenylboronic acid and TRIS-EDTA methods to confirm Amp C production. The phenylboronic acid method has identified 26 (59.09\%) as Amp C producers and TRISEDTA method has identified 18 (40.90\%) isolates as Amp C producers (Table 4). 
Table.1 Socio-demographic profile of study population $(n=200)$

\begin{tabular}{|c|c|c|}
\hline Age group & Frequency & Percent \\
\hline \multicolumn{2}{|c|}{ I. Age group } \\
\hline Up to 15 years & 12 & 6.0 \\
\hline 16 to 45 years & 99 & 49.5 \\
\hline 46 to 60 years & 45 & 22.5 \\
\hline Above 60 years & 44 & 100.0 \\
\hline Total & 200 & $27 \%$ \\
\hline Male & II.Gender & $73 \%$ \\
\hline
\end{tabular}

Table.2 Description of ESBL and Amp C AmpC production among the clinical isolates $(\mathrm{n}=200)$

\begin{tabular}{|c|c|c|c|c|}
\hline Parameter & \multirow{2}{*}{ Frequency } & \multirow{2}{*}{ percentage } & \multicolumn{2}{|c|}{ 95\% CI } \\
\cline { 4 - 5 } & & Lower & Upper \\
\hline $\begin{array}{c}\text { Number of Multidrug-resistant } \\
\text { stains }\end{array}$ & 94 & $47 \%$ & $40.08 \%$ & $53.92 \%$ \\
\hline $\begin{array}{c}\text { Number of ESBL producing } \\
\text { strains }\end{array}$ & 84 & $41 \%$ & $34.18 \%$ & $47.82 \%$ \\
\hline
\end{tabular}

Table.3 Description of ESBL and Amp C AmpC production among the clinical isolates ( $\mathrm{n}=84)$

\begin{tabular}{|l|c|c|c|c|}
\hline \multicolumn{1}{|c|}{ Parameter } & \multirow{2}{*}{ Frequency } & percentage & \multicolumn{2}{c|}{ 95\% CI } \\
\cline { 4 - 5 } & & Lower & Upper \\
\hline $\begin{array}{l}\text { Number of strains producing } \\
\text { AmpC by disk diffusion method }\end{array}$ & 44 & $52.38 \%$ & $41.7 \%$ & $63.06 \%$ \\
\hline $\begin{array}{l}\text { Number of ESBL and AmpC co- } \\
\text { producers by disk diffusion } \\
\text { method }\end{array}$ & 38 & $45.23 \%$ & $34.59 \%$ & $55.87 \%$ \\
\hline
\end{tabular}


Table.4 Description of ESBL and Amp C AmpC production among the clinical isolates $(n=44)$

\begin{tabular}{|l|c|c|}
\hline \multicolumn{1}{|c|}{ Parameter } & Frequency & percentage \\
\hline $\begin{array}{l}\text { Number of AmpC producing strains } \\
\text { by Phenyl boronic acid }\end{array}$ & 26 & $59.09 \%$ \\
\hline $\begin{array}{l}\text { Number of strains producing Amp C } \\
\text { by TRIS -EDTA }\end{array}$ & 18 & $40.90 \%$ \\
\hline
\end{tabular}

In recent times, many studies across the globe and India have reported a sharp rise in ESBL producing strains of $E$. coli. But there are wide variations across the settings in the reported prevalence of ESBL producing strains. Of late various studies across the world and also in India have reported a substantial increase in ESBL producing strains of E. coli. But in the prevalence of ESBL producing strains it has been reported that there are wide variations across the settings. Recently a study was carried out in a tertiary care teaching hospital in south India, and we found out in our study that there was a high percentage of ESBL isolates $(41 \%)$, but prior to our study some reporters have shown the rate of $23.83 \%$ by Sood et al., (2012). $34.42 \%$ by Akram et al., $38.5 \%$ by Soltani et al., and $31.3 \%$ by Jadhav et al., they have reported to be slightly lower when compared to the present study. Whereas some studies of Bajpai $e t$ al., Grover et al., etc. have reported to have a similar proportion $(41.6 \%$ and $42.2 \%$, respectively) of ESBL among urinary isolates of E. coli.

On the contrary, a much higher proportion of ESBL ranging from $53.4 \%$ have been reported by many other authors like by Ahmed et al., two-thirds by Eshwarappa et al., But on the whole, an important fact is being insisted by most of these studies is that a higher resistance pattern among these ESBL producing strains have been evolved when compared to non-ESBL producing strains.
The proportion of Amp $\mathrm{C}$ producers was $52.38 \%$ (41.7\% and $63.06 \%)$ among ESBL producing strains 4 and $45.23 \%$ (34.59\%) were ESBL and Amp C co-producers have been reported in the current study. Grover $e t$ al., have reported coexistence of ESBL and Amp C Beta-lactamase producers was detected in $9.9 \%(26 / 262)$ of the isolates. Only $9.2 \%$ of isolates to be Amp C producers and most of them to ESBL and Amp C co-producers have been reported by Hemalatha et al., in their study. By disc diffusion method, Phenylboronic acid and TRIS- EDTA yielded 59.09\% and 40.90\% positive results for Amp C production among 44 Amp $\mathrm{C}$ producers have reported Prevalence of Amp $\mathrm{C}$ production by inhibitor based method using boronic acid (IBM) at $40 \%$ and $39 \%$ and modified threedimensional test (M3D) respectively have been reported by Handa et al., Hassan et al., by standard three-dimensional enzyme extract assay have reported the proportion of Amp C producers to be $62.8 \%$, and also concluded that among various methods tested, boronic acid disk test to be was highly sensitive (88\%) and specific (92\%), compared to other methods. Saad et al., evaluated the sensitivity and specificity of disc disk approximation test compared to three-dimensional extract and reported a sensitivity of $88 \%$ and specificity of $92 \%$, with an overall diagnostic accuracy of $90.24 \%$. Thus a routine implementation of disc disk approximation methods for the evaluation of Amp $\mathrm{C}$ production is 
recommended pertaining to these finding findings.

In conclusion, simple methods to detect Amp C production, such as disc disk diffusion method and phenylboronic acid method are useful in identifying Amp C producing strains of $E$. coli.

The yield of these simple methods is comparable to more complicated methods like TRIS-EDTA.

\section{Recommendations}

Assessment of Amp C production by simple methods like phenylboronic acid should be part of routine evaluation in assessing antibiotic susceptibility of Multidrugresistant strains of E. coli. This can minimize the chances of false susceptibility reporting and can have a huge positive impact on treatment outcomes.

\section{References}

Aboumarzouk, O.M. 2014. Extended spectrum beta-lactamase urinary tract infections. Urol. annals, 6(2): 114-5.

Ahmed, I., Sajed, M., Sultan, A., Murtaza, I., Yousaf, S., Maqsood, B., et al. 2015. The erratic antibiotic susceptibility patterns of bacterial pathogens causing urinary tract infections. EXCLI J., 14: 916-25.

Akram, M., Shahid, M., Khan, A.U. 2007. Etiology and antibiotic resistance patterns of community-acquired urinary tract infections in $\mathrm{J} \mathrm{N} \mathrm{M} \mathrm{C}$ Hospital Aligarh, India. Annals of Clin. Microbiol. Antimicrobials, 6: 4.

Bajpai, T., Pandey, M., Varma, M., Bhatambare, G.S. 2014. Prevalence of extended spectrum beta-lactamase producing uropathogens and their antibiotic resistance profile in patients visiting a tertiary care hospital in central India: Implications on empiric therapy. Indian J. Pathol. Microbiol., 57(3): 407-12.

Drinkovic, D., Morris, A.J., Dyet, K., Bakker, S., Heffernan, H. 2015. Plasmid-mediated Amp C betalactamase-producing Escherichia coli causing urinary tract infection in the Auckland community likely to be resistant to commonly prescribed antimicrobials. The New Zealand Med. J., 128(1410): 50-9.

Eshwarappa, M., Dosegowda, R., Aprameya, I.V., Khan, M.W., Kumar, P.S., Kempegowda, P. 2011. Clinicomicrobiological profile of urinary tract infection in south India. Indian $J$. Nephrol., 21(1): 30-6.

Grover, N., Sahni, A.K., Bhattacharya, S. 2013. Therapeutic challenges of ESBLS and Amp C beta-lactamase producers in a tertiary care center. Med. J., Armed Forces India, 69(1): 410.

Gupta, V., Kumarasamy, K., Gulati, N., Garg, R., Krishnan, P., Chander, J. 2012. Amp C beta-lactamases in nosocomial isolates of Klebsiella pneumoniae from India. The Indian J. Med. Res., 136(2): 237-41.

Gupta, V., Rani, H., and Singla, N. 2013. Determination of Extended-Spectrum beta-Lactamases and Amp C Production in Uropathogenic Isolates of Escherichia coli and Susceptibility to Fosfomycin. J. Lab. Physicians, 5(2): 90-3.

Handa, D., Pandey, A., Asthana, A.K., Rawat, A., Handa, S., Thakuria, B. 2013. Evaluation of phenotypic tests for the detection of Amp C betalactamase in clinical isolates of Escherichia coli. Indian J. Pathol. Microbiol., 56(2):135-8. 
Harris, PN. 2015. Clinical management of infections caused by Enterobacteriaceae that express extended-spectrum beta-lactamase and Amp C enzymes. Seminars in respiratory and critical care medicine, 36(1):56-73.

Hassan, A., Usman, J., Kaleem, F., Gill, M.M., Khalid, A., Iqbal, M., et al. 2013. Evaluation of different phenotypic methods for detection of Amp C Beta-lactamase producing bacteria in clinical isolates. J. College of Physicians and SurgeonsPakistan, JCPSP, 23(9): 629-32.

Hemalatha, V., Padma, M., Sekar, U., Vinodh, T.M., Arunkumar, A.S. 2007. Detection of Amp C beta lactamases production in Escherichia coli \& Klebsiella by an inhibitor based method. The Indian J. Med. Res., 126(3): 220-3.

Jadhav, S., Hussain, A., Devi, S., Kumar, A., Parveen, S., Gandham, N., et al. 2011. Virulence characteristics and genetic affinities of multiple drug resistant uropathogenic Escherichia coli from a semi urban locality in India. PloS one, 6(3): e18063.

Jaurin, B., and Grundstrom, T. 1981. Amp C cephalosporinase of Escherichia coli K-12 has a different evolutionary origin from that of beta-lactamases of the penicillinase type. Proceedings of the National Academy of Sciences of the United States of America, 78(8): 4897-901.

Kumar, E., Usha, K., Chaudhury, A., Ramana, B.V., Gopal, D.V. Detection of Amp C beta-lactamases production in Acinetobacter species by inhibitor (disk) based \& modified three dimensional (enzyme extraction) methods. The Indian J. Med. Res., 140(5): 688-90.
Lina, T.T., Khajanchi, B.K., and Azmi, I.J. 2014. Phenotypic and molecular characterization of extended-spectrum beta-lactamase-producing Escherichia coli in Bangladesh. PloS one, 9(10): e108735.

Manoharan, A., Sugumar, M., Kumar, A., Jose, H., Mathai, D., Khilnani G.C. et al., 2012. Phenotypic \& molecular characterization of Amp C betalactamases among Escherichia coli, Klebsiella spp. \& Enterobacter spp. from five Indian Medical Centers. The Indian J. Med. Res., 135: 359-64.

Matsumura, Y., Yamamoto, M., Higuchi, T., Komori, T., Tsuboi, F., Hayashi A, et al., 2012. Prevalence of plasmidmediated Amp C beta-lactamaseproducing Escherichia coli and spread of the ST131 clone among extendedspectrum beta-lactamase-producing $E$. coli in Japan. Int. J. Antimicrobial Agents, 40(2):158-62.

Metri, B.C., Jyothi, P., and Peerapur, BV. 2012. Detection of ESBL in E. coli and $\mathrm{K}$. pneumoniae isolated from urinary tract infection. Indian $J$. Nephrol., 22(5): 401-2.

Mittal, S., Sharma, M., and Chaudhary, U. 2014. Study of virulence factors of uropathogenic Escherichia coli and its antibiotic susceptibility pattern. Indian J. Pathol. Microbiol., 57(1): 61-4.

Paltansing, S., Kraakman, M., van Boxtel, R., Kors, I., Wessels, E., Goessens, W et al., 2015. Increased expression levels of chromosomal Amp C betalactamase in clinical Escherichia coli isolates and their effect on susceptibility to extended-spectrum cephalosporins. Microbial drug resistance (Larchmont, NY).21(1):716.

Paniagua-Contreras, G.L., Monroy-Perez, E., and Rodriguez-Moctezuma, J.R. 2015. Virulence factors, antibiotic 
resistance phenotypes and $\mathrm{O}$ serogroups of Escherichia coli strains isolated from community-acquired urinary tract infection patients in Mexico. J. Microbiol. Immunol. Infect., xx, 1-8.

Rasamiravaka, T., Shaista Sheila,HS., and Rakotomavojaona.2015. Changing profile and increasing antimicrobial resistance of uropathogenic bacteria in Madagascar. Medecine et maladies infectieuses, 45(5):173-6.

Saad, N., Munir, T., Ansari, M., Gilani, M., Latif, M., Haroon, A. 2016. Evaluation of phenotypic tests for detection of Amp C beta-lactamases in clinical isolates from a tertiary care hospital of Rawalpindi, Pakistan. JPMA The J. Pakistan Med. Assoc., 66(6): 658-61.

Sageerabanoo, S., Malini, A., Mangaiyarkarasi, T., Hemalatha, G. Phenotypic detection of extended spectrum beta-lactamase and Amp-C beta-lactamase producing clinical isolates in a Tertiary Care Hospital: A preliminary study. J. Natural Sci. Biol. Med., 6(2): 383-7.

Shanthi, M., Sekar, U., Arunagiri, K., Sekar, B. 2012. Detection of Amp C genes encoding for beta-lactamases in Escherichia coli and Klebsiella pneumoniae. Indian J. Med. Microbiol., 30(3): 290-5.
Soltani, R., Ehsanpoor, M., Khorvash, F., Shokri, D. 2014. Antimicrobial susceptibility pattern of extendedspectrum beta-lactamase-producing bacteria causing nosocomial urinary tract infections in an Iranian referral teaching hospital. J. Res. pharmacy practice, 3(1): 6-11.

Sood, S., and Gupta, R. 2012. Antibiotic resistance pattern of community acquired uropathogens at a tertiary care hospital in jaipur, rajasthan. Indian journal of community medicine: official publication of Indian Association of Preventive \& Social Medicine, 37(1): 39-44.

Taneja, N., Rao, P., Arora, J., Dogra, A. 2008. Occurrence of ESBL \& Amp-C beta-lactamases \& susceptibility to newer antimicrobial agents in complicated UTI. The Indian J. Med. Res., 127(1): 85-8.

Tracz, D.M., Boyd, D.A., Hizon, R., Bryce, E., McGeer, A., Ofner-Agostini, M. et al., 2007. Amp C gene expression in promoter mutants of cefoxitinresistant Escherichia coli clinical isolates. FEMS Microbiol. Letters, 270(2):265-71.

Yang, K., Guglielmo, B.J. 2007. Diagnosis and treatment of extended-spectrum and Amp C beta-lactamase-producing organisms. The Annals of Pharmacother., 41(9): 1427-35.

\section{How to cite this article:}

Anandhi Lakshmanan, Dharmalingam Thirunavukkarasu, T.Umaarasu and S. Rajesh. 2016. Assessment of Amp C Beta-lactamase Production by Various Methods in Urinary Isolates of Escherichia coli from a Tertiary Care Teaching Hospital. Int.J.Curr.Microbiol.App.Sci. 5(11): 388-395. doi: http://dx.doi.org/10.20546/ijcmas.2016.511.044 